\title{
BRAIDS, HYPERGEOMETRIC FUNCTIONS, AND LATTICES
}

\author{
G. D. MOSTOW
}

1. Braids. Let $L_{1}, L_{2}$ be two parallel lines in the plane $y=0$ of $(x, y, z)$ space, $L_{1}$ at $z=r_{1}$ and $L_{2}$ at $z=r_{2}$. Let $P_{i}=\left(i, 0, r_{1}\right), Q_{i}=\left(i, 0, r_{2}\right)$, $i=1, \ldots, n$.

A braided n-path is a set of $n$ paths $c_{i}(t)$ in $\mathbf{R}^{3}(i=1, \ldots, n)$ satisfying

(1) $c_{i}(t)=\left(x_{i}(t), y_{i}(t), t\right), r_{1} \leqslant t \leqslant r_{2}, c_{i}\left(r_{1}\right)=P_{i}, c_{i}\left(r_{2}\right) \in\left\{Q_{1}, \ldots, Q_{n}\right\}$.

(2) The paths do not intersect.

Two braided $n$-paths are regarded as equivalent if and only if it is possible to deform the one configuration into the other respecting conditions (1) and (2) throughout the deformation; note that one does permit $r_{1}, r_{2}$ to vary so long as $r_{1}<r_{2}$ is respected. Thus (a) and (b) in Figure 1 represent the same braid. By definition, a braid is an equivalence class of braided $n$-paths.

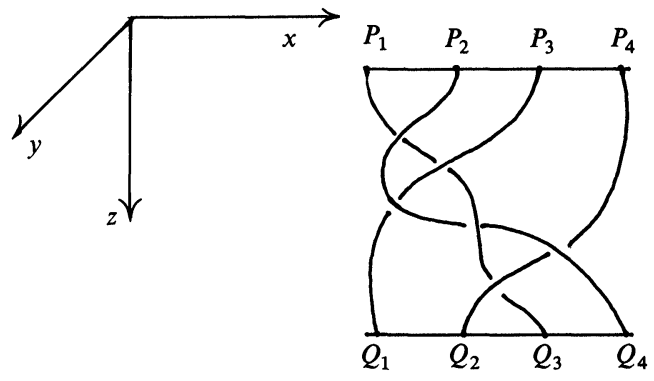

(a)

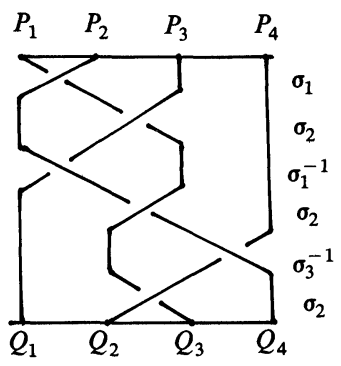

(b) $\sigma_{2} \sigma_{3}^{-1} \sigma_{2} \sigma_{1}^{-1} \sigma_{2} \sigma_{1}$

FIGURE 1.

Two braids $A$ and $B$ can be multiplied: $B \cdot A$ is the braid obtained by first braiding $A$ then $B$, and adjusting the domain of the parameter $t$ so that it changes without interruption, i.e., by bringing the end line of $A$ and initial line of $B$ together and then erasing them.

Received by the editors May 20, 1986.

1980 Mathematics Subject Classification (1985 Revision). Primary 06B30, 20F36, 33A30.

This paper is based partly on my 1985 Jacqueline Lewis Lectures at Rutgers University and Sackler Lectures at Tel Aviv University. The research was supported in part by NSF Grant DMS-8506130. 


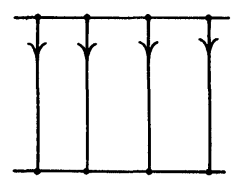

(a)

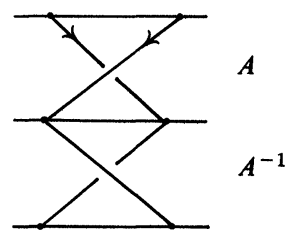

(b) $A^{-1} \cdot A=$ identity

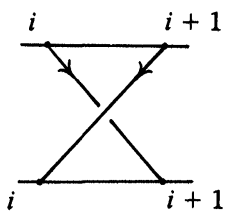

(c)

FIGURE 2

Multiplication of braids is associative. The braid in which no paths intertwine (Figure 2(a)) is the identity braid. The inverse of a braid $A$ with path $c^{A}$ is defined by its mirror image (Figure 2(b)), i.e., in the inverse of $A, c_{i}^{A^{-1}}(t)=$ $c_{i}^{A}(-t),-r_{2} \leqslant t \leqslant-r_{1}$. Thus, under multiplication, the braids form a group. We denote by $\sigma_{i}$ the braid which joins $i$ to $i+1$ (Figure 2(c)) by a path passing under the path joining $i+1$ to $i$. Then $\left\{\sigma_{1}, \ldots, \sigma_{n-1}\right\}$ generate the braid group on $n$-strings in $\mathbf{R}^{3}$; we denote this group $B_{n}$. It is referred to in the literature as the "algebraic braid group."

Each braid $b$ in $B_{n}$ effects a permutation $\bar{b}$ of $\{1, \ldots, n\}$. The map $\pi: b \rightarrow \bar{b}$ is a homomorphism of $B_{n}$ onto $\Sigma_{n}$, the permutation group on $n$ letters. Let

$$
C_{n}=\operatorname{Ker} \pi \text {. }
$$

$C_{n}$ is called the "colored braid group" or "pure braid group." The paths defining any braid $b$ in $C_{n}$ go from each point $i$ on the initial line $L_{1}$ of $b$ to a point $i$ in the end line $L_{2}$ of $b$. The name of $C_{n}$ comes from the idea of coloring each point $P_{1}, \ldots, P_{n}$ a separate color and coloring the path going from $P_{i}$ to $Q_{i}$ with the $i$ th color $(i=1, \ldots, n)$.

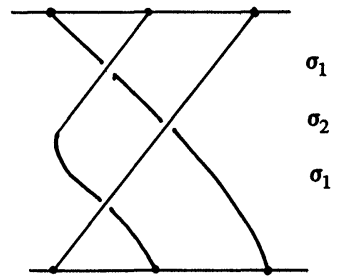

(a)

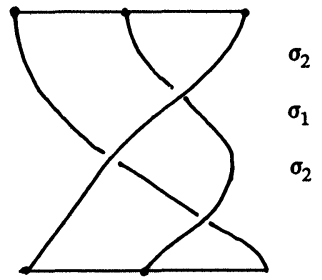

(b)

$$
\sigma_{1} \sigma_{2} \sigma_{1}=\sigma_{2} \sigma_{1} \sigma_{2}
$$

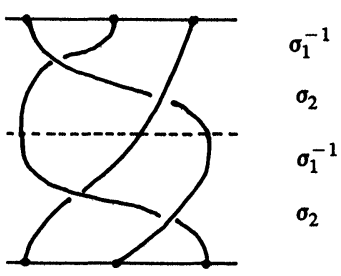

(c)

A girl's braid $=\left(\sigma_{2} \sigma_{1}^{-1}\right)^{n}$.

FIGURE 3

The braid group $B_{n}$ was first defined by Emil Artin in Theorie der Zöpfe, Abhandlungen aus der Math. Seminar der Hamburg Universität 4 (1925). He proved there that $B_{n}$ has the presentation

Generators: $\sigma_{1}, \sigma_{2}, \ldots, \sigma_{n-1}$.

Relations: $\quad \sigma_{i} \sigma_{j}=\sigma_{j} \sigma_{i}$ for $|i-j|>1$,

$$
\sigma_{i} \sigma_{i+1} \sigma_{i}=\sigma_{i+1} \sigma_{i} \sigma_{i+1} \quad(i=1, \ldots, n-2) \text {. }
$$

Let $a=\sigma_{1} \sigma_{2} \cdots \sigma_{n-1}$. We have

$$
a \sigma_{1}=\sigma_{1} \cdots \sigma_{n-1} \sigma_{1}=\sigma_{1} \sigma_{2} \sigma_{1} \sigma_{3} \sigma_{4} \cdots \sigma_{n-1}=\sigma_{2} \sigma_{1} \sigma_{2} \sigma_{3} \sigma_{4} \cdots \sigma_{n-1}=\sigma_{2} \cdot a .
$$

Similarly $a \sigma_{i} a^{-1}=\sigma_{i+1}(i=1,2, \ldots, n-2)$. Thus $\left\{\sigma_{1}, a\right\}$ generate $B_{n}$. 


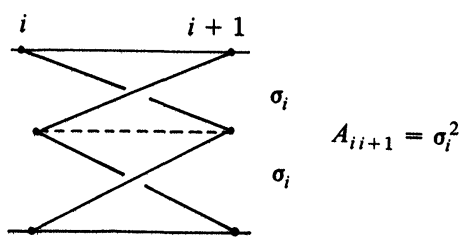

FIGURE 4

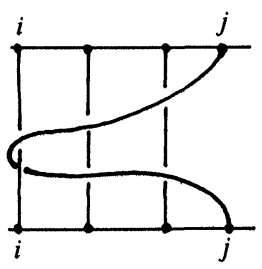

FIGURE 5

In his paper Theory of braids [1] Artin gives a presentation for the colored braid group $C_{n}$.

For any $i<j$, set $A_{i j}=A_{j i}=\sigma_{j-1}^{-1} \sigma_{j-2}^{-1} \cdots \sigma_{i+1}^{-1} \sigma_{i}^{2} \sigma_{i+1} \cdots \sigma_{j-2} \sigma_{j-1}$.

Generators for $C_{n}:\left\{A_{i j} ; 1 \leqslant i<j<n\right\}$.

Relations: 1. $A_{r s} A_{i j} A_{r s}^{-1}=A_{i j}$ if $r<s<i<j$ or $i<r<s<j$, i.e., if $(r, s)$ and $(i, j)$ do not each separate the other. If $(r, s)$ and $(i, j)$ do separate each other, choose $i<r<s$ or any even permutation of such $i, r, s$. Then

2. $A_{r s}^{-1} A_{i r} A_{r s}=A_{i s} A_{i r} A_{i s}^{-1}$.

3. $A_{r s}^{-1} A_{i s} A_{r s}=A_{i s} A_{i r} A_{i s}\left(A_{i s} A_{i r}\right)^{-1}$.

If $i<r<j<s$, then

4. $A_{r s}^{-1} A_{i j} A_{r s}=\left(A_{i r} A_{i s} A_{i r}^{-1} A_{i s}^{-1}\right) A_{i j}\left(A_{i r} A_{i s} A_{i r}^{-1} A_{i s}^{-1}\right)^{-1}$.

It is a topological fact that

THEOREM. Given any braided n-path $\left\{c_{i}(t) ; i=1,2, \ldots, n\right\}$ there is a continuous family of homeomorphisms $\left\{\varphi_{t} ; 0 \leqslant t \leqslant 1\right\}$ of $\mathbf{R}^{2}$ onto $\mathbf{R}^{2}$ such that

$$
c_{i}(t)=\left(\varphi_{t}\left(c_{i}(0)\right), t\right) \quad 0 \leqslant t \leqslant 1,1 \leqslant i \leqslant n .
$$

That is, a braided $n$-path can be regarded as a deformation of $n$ distinct points $c_{1}(0), \ldots, c_{n}(0)$ in $\mathbf{R}^{2}$, and this deformation can be extended to an isotopy $\left\{\varphi_{t} ; 0 \leqslant t \leqslant 1\right\}$ of $\mathbf{R}^{2}$. This isotopy is of course not unique, but any two such isotopies can be deformed into one another.

Our discussion of braids can be slightly generalized in two ways.

1. There is no need to restrict the endpoints of the $n$-stringed braids to lie in the plane $y=0$; the $n$ points can be taken anywhere in the plane $\mathbf{R}^{2}$.

2 . We can consider $n$-string braids whose endpoints lie anywhere on the 2-sphere $S^{2}=\mathbf{R}^{2} \cup \infty$. In that case the deformations can take place in $S^{2} \times \mathbf{R}$ rather than $\mathbf{R}^{2} \times \mathbf{R}$. We distinguish this braid group from the previous one by denoting them $B_{n}\left(S^{2}\right)$ and $B_{n}\left(\mathbf{R}^{2}\right)$ respectively.

Clearly any $n$-string braid in $\mathbf{R}^{2}$ can be regarded as a braid in $S^{2}$ and so there is a natural map $B_{n}\left(\mathbf{R}^{2}\right) \rightarrow B_{n}\left(S^{2}\right)$; it is a homomorphism and its kernel is the normal subgroup generated by the element $\sigma_{1} \cdots \sigma_{n-2} \sigma_{n-1}^{2} \sigma_{n-2} \cdots \sigma_{1}$.
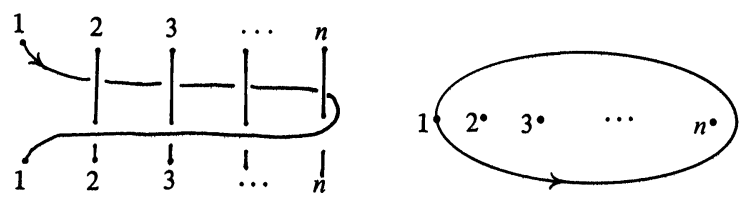

FIGURE 6(a) 
Next consider the pair $(P, S)$ where $P=\mathrm{C} \cup \infty$, the Riemann sphere, i.e., complex projective 1-dimensional space, and $S=\left\{s_{0}, s_{1}, \ldots, s_{n-1}\right\}$ is a set of $n$ points in $P$. We can associate to any element of the $n$-string braid group $B_{n}(P)$ an isotopy of $P$ which moves $S$ around in $P$ and finally brings it back to its original position (permuted). Thus any braid in $B_{n}(P)$ will induce a well-defined action on any structure associated with $(P, S)$ whose objects do not change under deformations fixing the points of $S$. We give three examples.

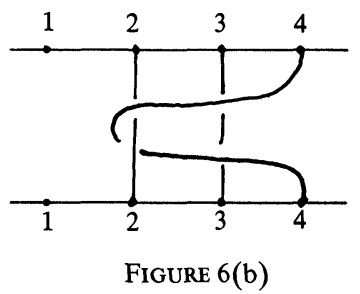

(a)

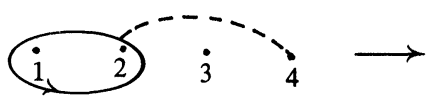

(c)

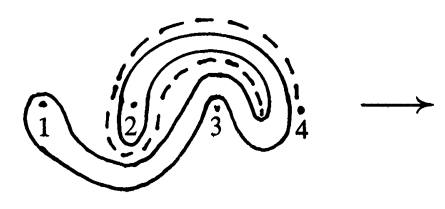

(b)

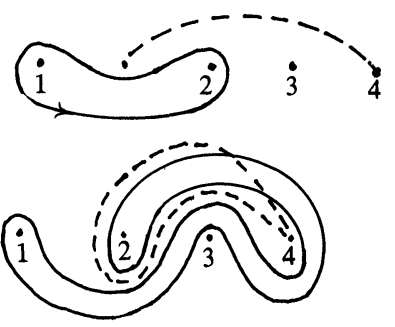

FIGURE 7

EXAMPLE 1. $B_{n}(P)$ acts on $\pi_{1}(P-S)$, the fundamental group of the space $P-S$. Consider the effect of the braid $A_{24}$ (Figure 6(b)) on the path around 1 and 2 (Figure 7).

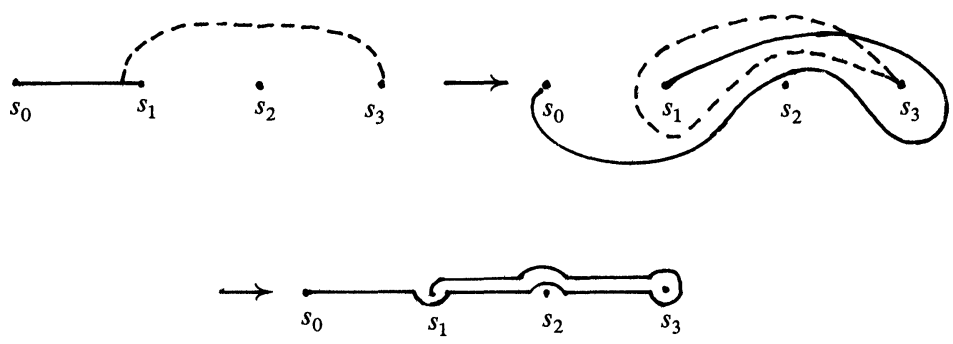

Figure 8

EXAMPle 2. $B_{4}(P)$ acts on $H_{1}(P, S)$ (Figure 8).

EXAMPLE 3. Let

$$
f_{i j}\left(s_{0}, s_{1}, \ldots, s_{n-1}\right)=\int_{s_{i}}^{s_{j}}\left(z-s_{0}\right)^{-\mu_{0}}\left(z-s_{1}\right)^{-\mu_{1}} \cdots\left(z-s_{n-1}\right)^{-\mu_{n-1}} d z
$$


where $S=\left\{s_{0}, \ldots, s_{n-1}\right\}, \mu_{0}, \ldots, \mu_{n-1}$ are complex numbers, and the path of integration is selected in $P-S$. Although the integrand is multivalued, the value of $f_{i j}$ does not change when the path of integration from $s_{i}$ to $s_{j}$ is deformed in $P-S$, so long as the elements of $S$ are fixed. Hence the colored braid group $C_{n}(P)$ will act on the linear span of the multivalued functions $\left\{f_{i j}: 0 \leqslant i<j \leqslant n-1\right\}$. Indeed the full braid group $B_{n}(P)$ will act if $\mu_{i}=\mu_{j}$ for all $i, j$. For example, if $n=3$, and the path of integration from $s_{i}$ to $s_{i+1}$ is a straight line $(i=0,1,2)$ then the effect of $A_{24}$ on $f_{01}$ is a result of two changes: 2.

(i) One is the effect on the path of integration; this is illustrated in Example

(ii) Since the new path of integration from $s_{0}$ to $s_{1}$ makes a loop around $s_{3}$, the integrand changes by a factor $e^{-2 \pi i \mu_{3}}$ for the integration from $s_{3}$ to $s_{1}$. Hence

$$
\begin{aligned}
A_{24} f_{01} & =f_{01}+f_{12}+f_{23}+e^{-2 \pi i \mu_{3}}\left(-f_{23}-f_{12}\right) \\
& =f_{01}-\left(e^{-2 \pi i \mu_{3}}-1\right)\left(f_{12}+f_{23}\right) .
\end{aligned}
$$

Let $\mu=\left(\mu_{0}, \mu_{1}, \ldots, \mu_{n-1}\right)$ and let $V_{\mu}$ denote the linear span of the functions $\left\{f_{i j} ; 1 \leqslant i<j \leqslant n-1\right\}$. By the argument used in Example 3, we see that $C_{n}(P)$ acts on $V_{\mu}$ and sends $V_{\mu}$ to itself. One obtains thereby a homomorphism of the colored braid group into the group of linear automorphisms of the vector space $V_{\mu}$; the image of $C_{n}(P)$ is denoted $\Gamma_{\mu}$ and is called the monodromy group of $V_{\mu}$.

2. Schwarz's Problems. The next example relates the monodromy action of the algebraic braid group to a classical result of H. A. Schwarz.

EXAMPLE 4. Let $\omega=z^{-\mu_{0}}(z-1)^{-\mu_{1}}(z-x)^{-\mu_{x}} d z$. Set $u=z^{-1}$. Then

$$
\begin{aligned}
\int_{1}^{\infty} & \omega=\int_{1}^{0} u^{\mu_{0}}\left(\frac{1-u}{u}\right)^{-\mu_{1}}\left(\frac{1-u x}{u}\right)^{-\mu_{x}}\left(-\frac{1}{u^{2}}\right) d u \\
& =\int_{0}^{1} u^{\mu_{0}+\mu_{1}+\mu_{x}-2}(1-u)^{-\mu_{1}}(1-u x)^{-\mu_{x}} d u \\
& =\int_{0}^{1} u^{-\mu_{\infty}}(1-u)^{-\mu_{1}}(1-u x)^{-\mu_{x}} d u, \text { where } \mu_{\infty}=2-\mu_{0}-\mu_{1}-\mu_{x} .
\end{aligned}
$$

In 1778, Euler introduced the hypergeometric series

$$
F(a, b, c ; x)=\sum \frac{(a, n)(b, n)}{(c, n)} \frac{x^{n}}{n !}, \quad|x|<1,
$$

where $(a, n)=\prod_{i=0}^{n-1}(a+i)$, as a solution of the hypergeometric differential equation

$$
x(1-x) y^{\prime \prime}+(c-(a+b+1) x) y^{\prime}-a b y=0
$$

and he knew the identity

$$
\frac{\Gamma(b) \Gamma(c-b)}{\Gamma(c)} F(a, b, c ; x)=\int_{0}^{1} u^{b-1}(1-u)^{c-b-1}(1-u x)^{-a} d u .
$$


Thus $\mu_{\infty}=1-b, \mu_{1}=1+b-c, \mu_{x}=a$, and

$$
\int_{1}^{\infty} \omega=\text { constant } \times F\left(\mu_{x}, 1-\mu_{\infty}, 2-\mu_{1}-\mu_{\infty} ; x\right) .
$$

It was observed by Pochhammer (1870) and Schläfli (1871) that, for any $g, h \in S=\{0,1, x, \infty\}$, the integral $\int_{g}^{h} \omega$ is a multivalued function of $x$ satisfying (HDE). H. A. Schwarz, in his seminal paper Über diejenige Fälle in welchen die Gaussische hypergeometrische Reihe eine algebraische Function ihres viertes elementes darstellt, J. für Math. (1873), considered the space $W$ of all solutions of (HDE). Each solution is multivalued on $P-\{0,1, x, \infty\}$ and under analytic continuation around the punctures $S=\{0,1, x, \infty\}$, each solution goes into another element of $W$. Take as a base in $W$

$$
F_{1}(x)=\int_{0}^{1} \omega, \quad F_{2}(x)=\int_{1}^{x} \omega .
$$

The effect of moving $x$ around 1 on $F_{1}(x)$ is given as in Example 2 above by the change in the path of integration from (a) to (b) in Figure 9 and the change in the value of the integrand. Thus $F_{1}(x) \rightarrow F_{1}(x)+F_{2}(x)-e^{-2 \pi i \mu_{x}} F_{2}(x)$ and Schwarz's space $W$ coincides with our $V_{\mu}$, where $\mu_{0}=c-a, \mu_{1}=1+b$ $-c, \mu_{x}=a$.

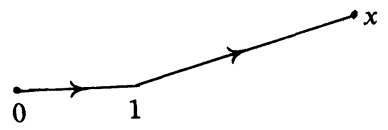

(a) $\dot{\infty}$

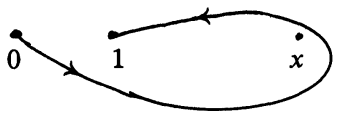

(b)

FIGURE 9

Classically, this change under analytic continuation is called "monodromy of $x$ around 1," and the set of all monodromies arising from moving one puncture in $S$ around another generates the monodromy group. It is nothing but our previously defined monodromy action of $C_{4}(P)$ on $V_{\mu}$.

The hypergeometric function $F_{1}(x)$ is an algebraic function of $x$ if and only if the monodromy group $\Gamma_{\mu}$ is a finite group. For this to happen, one needs $0<\mu_{s}<1$ for all $s \in S, \mu_{0}+\mu_{1}+\mu_{x}<1$, and some additional conditions related to the regular platonic solids. Schwarz determined all these cases (cf. Figure 10; $\pi \lambda_{x}=$ angle at $\left.f(x), x \in\{0,1, \infty\}\right)$.

In the same paper Schwarz considers the case of infinite monodromy groups and gives sufficient conditions for the monodromy group to be discrete. To answer this question, it suffices to consider the action of the monodromy group on the one-dimension projective space $P$ consisting of 1 -dimensional subspaces of $W$; let $\Gamma_{\mu}$ denote this monodromy group action on $P$. Accordingly, Schwarz considers the map

$$
f: x \rightarrow F_{1}(x) / F_{2}(x)
$$




\begin{tabular}{r|ccccl} 
& $\lambda_{0}$ & $\lambda_{1}$ & $\lambda_{\infty}$ & area $/ \pi$ & related polyhedron \\
\hline 1 & $\frac{1}{2}$ & $\frac{1}{2}$ & $\frac{1}{n}$ & & double pyramid \\
2 & $\frac{1}{2}$ & $\frac{1}{3}$ & $\frac{1}{3}$ & $\frac{1}{6}=A$ & tetrahedron \\
3 & $\frac{2}{3}$ & $\frac{1}{3}$ & $\frac{1}{3}$ & $\frac{1}{3}=2 A$ & \\
4 & $\frac{1}{2}$ & $\frac{1}{3}$ & $\frac{1}{4}$ & $\frac{1}{12}=B$ & cube or octahedron \\
5 & $\frac{2}{3}$ & $\frac{1}{4}$ & $\frac{1}{4}$ & $\frac{1}{6}=2 B$ & \\
6 & $\frac{1}{2}$ & $\frac{1}{3}$ & $\frac{1}{5}$ & $\frac{1}{30}=C$ & \\
7 & $\frac{2}{5}$ & $\frac{1}{3}$ & $\frac{1}{3}$ & $2 C$ & \\
8 & $\frac{2}{3}$ & $\frac{1}{5}$ & $\frac{1}{5}$ & $2 C$ & \\
9 & $\frac{1}{2}$ & $\frac{2}{5}$ & $\frac{1}{5}$ & $3 C$ & \\
10 & $\frac{3}{5}$ & $\frac{1}{3}$ & $\frac{1}{5}$ & $4 C$ & duodecahedron \\
11 & $\frac{2}{5}$ & $\frac{2}{5}$ & $\frac{2}{5}$ & $6 C$ & \\
12 & $\frac{2}{3}$ & $\frac{1}{3}$ & $\frac{1}{5}$ & $6 C$ & icosahedron \\
13 & $\frac{4}{5}$ & $\frac{1}{5}$ & $\frac{1}{5}$ & $6 C$ & \\
14 & $\frac{1}{2}$ & $\frac{2}{5}$ & $\frac{1}{3}$ & $7 C$ & \\
15 & $\frac{3}{5}$ & $\frac{2}{7}$ & $\frac{1}{3}$ & $10 C$ &
\end{tabular}

Figure 10. Schwarz's list

where $F_{1}, F_{2}$ are any two linearly independent elements of $W$. We assume that $\mu_{s}$ is real for all $s \in S$. The geometric picture that Schwarz unveils is very pretty:

$f$ maps the upper half-plane onto a triangle $T$ in $P$ bounded by circular arcs with angles $\pi \lambda_{0}, \pi \lambda_{1}, \pi \lambda_{\infty}$ at $f(0), f(1), f(\infty)$ respectively, and $f$ maps $\mathbf{C}$ minus the slit $[0 \leqslant x<\infty]$ to the quadrilateral $\mathscr{F}$ obtained by adjoining to the triangle $T$ its "Schwarz reflection" in the arc $f(0)$ to $f(\infty)$ (cf. Figure 11). If $\lambda_{0}+\lambda_{1}+\lambda_{\infty}<1$, then the three circular arcs of the triangle $T$ each meet a common circle $C$ orthogonally. The monodromy of $f$ around a puncture $s \in S$ induces a rotation of angle $2 \pi \lambda_{s}$ around $f(s)$. One has $\lambda_{s}=1-\mu_{s}-\mu_{x}$ for any $s \in\{0,1, \infty\}$. Schwarz proved: When

$$
\begin{aligned}
& \lambda_{0}^{-1}, \lambda_{1}^{-1}, \lambda_{\infty}^{-1} \text { are integers and } \\
& \lambda_{0}+\lambda_{1}+\lambda_{\infty}<1
\end{aligned}
$$
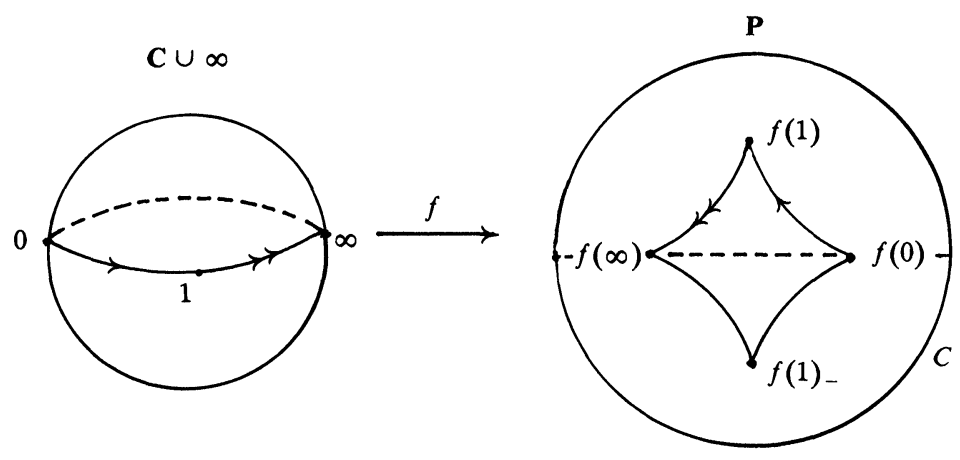

Figure 11 
then the effect of the monodromy group on the closure of the quadrilateral $\mathscr{F}=f(\mathbf{C}-[0 \leqslant x<\infty])$ is to move it over the entire interior of the disc $B^{1}$ bounded by $C$ so as to "tile" $B^{1}$ by quadrilaterals. Under these hypotheses the monodromy group acts discontinuously on the disc $B^{1}$, i.e., for any compact subset $K \subset B^{1}$ there is at most a finite set of distinct elements $\gamma$ of the monodromy group such that $\gamma K$ meets $K$. One can infer that the monodromy subgroup in Aut $W$ as well as $\Gamma_{\mu}$ in Aut $P$ are discrete.

Indeed, something even stronger is true when condition (INT) holds: The multivalued mapping $f: \mathbf{C} \cup \infty \rightarrow B^{1}$ has a single-valued inverse; that is, we can interpret $f$ as mapping a branched covering space of $\mathbf{C} \cup \infty$ onto the disc $B^{1}$ and $f$ has an analytic inverse $\pi: B \rightarrow \mathbf{C} \cup \infty$ such that $\pi(f(x))=x$ for all $x \in \mathbf{C} \cup \infty$. We can thus identify $\mathbf{C} \cup \infty$ with the orbit space $\Gamma_{\mu} \backslash B^{1}$ and $\pi$ as the natural projection $B^{1} \rightarrow \Gamma_{\mu} \backslash B^{1}$. One visualizes this geometrically by identifying the $\operatorname{arcs}[f(0), f(1)] \cong\left[f(0), f(1)_{-}\right],[f(1), f(\infty)] \cong\left[f(1)_{-}, f(\infty)\right]$.

There is an identity in the colored braid group on 4 strings relating the winding of $x$ around 0,1 , and $\infty$, which we denote $A_{0 x}, A_{1 x}, A_{\infty x}$ respectively; namely, $A_{0 x} A_{1 x} A_{\infty x}$ commutes with each of $A_{0 x}, A_{1 x}, A_{\infty x}$. Inasmuch as any bianalytic map of the disc $B^{1}$ commuting with rotations around the three distinct points $f(0), f(1), f(\infty)$ is the identity, we infer that $A_{\infty x}^{-1}=$ $A_{0 x} A_{1 x}$. This identity can also be deduced from the geometry of the quadrilateral $\mathscr{F}$.

The group Aut $B^{1}$ of bianalytic maps of $B^{1}$ onto $B^{1}$ is isomorphic to the group of bianalytic maps of the upper half-plane $H$ in $\mathbf{C} \cup \infty$, since there is a bianalytic mapping taking $B^{1}$ to $H$. Thus Aut $B^{1} \cong$ Aut $H$, and the latter is given by the group of all fractional linear transformations

$$
\frac{a z+b}{c z+d}, \quad z \in \mathbf{C} \cup \infty, \quad a, b, c, d \text { real. }
$$

Thus Aut $H$ is isomorphic to the "projective linear" group $\operatorname{PGL}(2, \mathbf{R})$, the group of all $2 \times 2$ real invertible matrices divided by the central subgroup consisting of scalar multiples of the identity. The group $G=\operatorname{PGL}(2, \mathbf{R})$ depends on three real parameters. Each subgroup $\Gamma_{\mu}$ with $\mu$ satisfying condition (INT) above is a discrete subgroup of $G$. From the fact that $\Gamma_{\mu} \backslash B^{1}$ is compact, we can deduce that $G / \Gamma_{\mu}$ is a compact space in its natural topology.

A subgroup $\Gamma$ of a topological group $G$ such that

(1) $\Gamma$ is discrete in $G$,

(2) $G / \Gamma_{\mu}$ is compact (or, more generally, has finite Haar measure),

is called a lattice in $G$. What we have shown above is that lattices in Aut $B^{1}$ ( $\cong \mathrm{PGL}(2, \mathbf{R})$ ) can be constructed as homomorphic images of the braid group on 4 strings.

More precisely, in both the first Schwarz case of finite groups acting on the Riemann sphere $\mathbf{P}^{1}=\mathbf{C} \cup \infty$, and in the second case of lattices operating on the disc $B^{1}$, the groups are homomorphic images of the colored braid group on 4 strings. In both cases the monodromy groups preserve a hermitian form, definite in the case where $\Gamma_{\mu}$ is finite, indefinite in the case that $\Gamma_{\mu}$ is infinite. 
In the paper Monodromy of hypergeometric functions and non-lattice integral monodromy by Deligne and Mostow [3], the Schwarz construction of monodromy groups is generalized to higher dimensions, and it turns out that for topological reasons, an hermitian form is preserved. Generalizing Schwarz's results then gives rise to two problems.

PROBLEM 1 . When is the monodromy group discrete?

Note that in the case that a definite hermitian form is preserved, the monodromy group is finite whenever it is discrete.

Problem 2. In the case that the (multivalued) hypergeometric functions of $n$ variables map the space of $n$ complex variables into the $n$-ball $B^{n}$, when does there exist a single-valued inverse?

The answers to these questions are worked out in $[3,10$, and 11] and will be described below.

\section{Schwarz's second problem in $n$ dimensions.}

3.1. In the previous section, we defined the action of the monodromy group $\Gamma_{\mu}$ on the 2-dimensional vector space of solutions of the (HDE)

$$
x(x-1) y^{\prime \prime}+(c-(a+b+1) x) y^{\prime}-a b y=0 .
$$

We also saw that the effect of analytic continuation of $x$ around 0,1 , or $\infty$ on solutions of the form

$$
\int_{s_{i}}^{s_{j}} z^{-\mu_{0}}(z-1)^{-\mu_{1}}(z-x)^{-\mu_{2}} d z
$$

where $s_{i}, s_{j} \in S=\{0,1, x, \infty\}$ could be computed knowing

(i) the action of the braid group on the homology group $H_{1}(P, S)$, and

(ii) the multiplier $e^{-2 \pi \sqrt{-1} \mu_{i}}$ on the integrand when $x$ makes a circuit about one of the elements $s_{i}$ of $S$.

This suggests that one can study the monodromy action in hypergeometric functions without resorting to differential equations, as was done historically, in dimension 1 by Schwarz and dimension 2 by E. Picard in 1885 ([13]; cf. also \$3.14 below).

Picard defined a hypergeometric function of two variables as a linear combination of functions of the type

$$
F_{s t}(x, y)=\int_{s}^{t} z^{-\mu_{0}}(z-1)^{-\mu_{1}}(z-x)^{-\mu_{2}}(z-y)^{-\mu_{3}} d z
$$

where $s, t \in\{0,1, x, y, \infty\}$ and one defines a linear combination of

$$
\begin{aligned}
F_{s t}\left(x_{2}, \ldots, x_{n+1}\right) & \\
& =\int_{s}^{t}\left(z-x_{0}\right)^{-\mu_{0}}\left(z-x_{1}\right)^{-\mu_{1}}\left(z-x_{2}\right)^{-\mu_{2}} \cdots\left(z-x_{n+1}\right)^{-\mu_{n+1}} d z,
\end{aligned}
$$

where $x_{0}=0, x_{1}=1, s, t \in\left\{x_{0}, \ldots, x_{n+1}, \infty\right\}$, to be a hypergeometric function of $n$ variables. Note that one can allow $x_{0}, x_{1}$ to be arbitrary without changing the class of functions defined since $(0,1, \infty)$ goes to $\left(x_{0}, x_{1}, \infty\right)$ by the change of variable $z \rightarrow a z+b$ with $b=x_{0}, a=x_{1}-x_{0}$.

The account presented here, taken from the joint paper with P. Deligne [3], studies the monodromy of the hypergeometric functions via the concept of flat vector bundles and cohomology with coefficients in a flat vector bundle. 
3.2. Let $P=\mathrm{C} \cup \infty$, the complex projective line.

$$
M=\left\{\left(x_{0}, \ldots, x_{n+2}\right) \in P^{n+3} ; x_{i} \neq x_{j} \text { for } i \neq j\right\} .
$$

For $m \in M$, set $P_{m}=P-\left\{x_{0}, \ldots, x_{n+2}\right\}$, where $m=\left(x_{0}, \ldots, x_{n+2}\right)$, and

$$
P_{M}=\left\{(x, m) \in P \times M, x \in P_{m}\right\} .
$$

$P_{M}$ is the family of $(n+3)$-punctured projective lines.

Fix an $\alpha \in \mathbf{C}^{n+3}, \alpha=\left(\alpha_{0}, \alpha_{1}, \ldots, \alpha_{n+2}\right)$, such that

$$
\begin{aligned}
& \alpha_{i} \neq 0, \quad 0 \leqslant i \leqslant n+2, \\
& \prod_{0}^{n+2} \alpha_{i}=1 .
\end{aligned}
$$

(Note: ultimately, we shall choose $\alpha_{i}=e^{2 \pi \sqrt{-1} \mu_{i}}$.)

Let $\hat{P}_{m}=$ the simply covering space of $P_{m}$ and let $\pi_{1}\left(P_{m}\right)$ denote the fundamental group of $P_{m} \cdot \pi_{1}\left(P_{m}\right)$ operates on $\hat{P}_{m}$ as the group of covering transformations and $P_{m}=\pi_{1}\left(P_{m}\right) \backslash \hat{P}_{m}$.

Let $\bar{\alpha}$ denote the homomorphism $\pi_{1}\left(P_{m}\right) \rightarrow \mathrm{C}-\{0\}$ which maps each "small" loop around $x_{i}$ (i.e., not looping around any $x_{j}$ for $j \neq i$ ) in the counter-clockwise sense to the complex number $\alpha_{i}$. Then set

$$
L_{m}=\mathrm{C} \times_{\pi_{1}\left(P_{m}\right)} \hat{P}_{m}:=\mathrm{C} \times \hat{P}_{m} / \mathscr{R},
$$

where $\mathscr{R}$ is defined as the relation on $\mathbf{C} \times \hat{P}_{m}$ :

$$
(c, l p) \equiv(c \bar{\alpha}(l), p) \quad \text { for all } l \in \pi_{1}\left(P_{m}\right) .
$$

The projection of $\mathbf{C} \times \hat{P}_{m}$ to $\hat{P}_{m}$ induces a projection of $L_{m}$ to $P_{m}$ with fiber $\mathbf{C}$ and as we move from fiber to fiber along a path $l$ in the punctured complex line $P_{m}$, the fiber $\mathbf{C}$ returns to itself, but multiplied by $\bar{\alpha}(l)$. Thus the piece of $L_{m}$ over a simply connected neighborhood $U$ in $P_{m}$ resembles $\mathbf{C} \times U$, but $L_{m} \neq \mathbf{C} \times P_{m}$. We call $L_{m}$ a flat one-dimensional vector bundle with monodromy $\alpha$ on $P_{m}$.

By similar considerations, one can construct a flat one-dimensional vector bundle $L$ on $P_{M}$ with the property:

\section{$L_{m}$ has monodromy $\alpha$ along each $P_{m}$,}

where $L_{m}$ denotes the restriction of $L$ to $P_{m} \cdot H^{1}\left(P_{m}, L_{m}\right)$, the cohomology group with coefficients $L_{m}$, is a vector space over $\mathbf{C}$; we shall say more about this later.

The projection $P_{M} \rightarrow M$ is, locally on $M$, a topological direct product. Hence the family $\left\{H^{1}\left(P_{m}, L_{m}\right) ; m \in M\right\}$ forms a flat vector bundle over $M$. This bundle is not quite canonically determined by $\alpha$ because $L_{m}$ is not; $L_{m}$ is merely uniquely determined up to an isomorphism $c \rightarrow \lambda c, \lambda \in \mathbf{C}^{*}:=\mathbf{C}-$ $\{0\}$.

We get a bundle canonically determined by $\alpha$ when we divide by $C^{*}$ and descend from each vector space $H^{1}\left(P_{m}, L_{m}\right)$ to the associated projective space. Set

$$
B(\alpha)_{M}=\left\{P H^{1}\left(P_{m}, L_{m}\right) ; m \in M\right\}
$$

this is a flat bundle of projective spaces. That is, fix a base point 0 in $M$, then

$$
B(\alpha)_{M}=P H^{1}\left(P_{0}, L_{0}\right) \times_{\pi_{1}(M, 0)} \hat{P}_{0} .
$$


The action of $\pi_{1}(M, 0)$ on $P H^{1}\left(P_{0}, L_{0}\right)$ can be described in two equivalent ways.

(i) Let $m(t)$ be a closed path in $M$ with $m(0)=m(1)=0$. As $P_{0}$ moves continuously in $P_{M}$ to position $P_{m(t)}, P H^{1}\left(P_{0}, L_{0}\right)$ gets transported via flatness in $B(\alpha)_{M}$ (i.e., horizontally) to $P H^{1}\left(P_{m}, L_{m}\right)$ and returns finally to $P H^{1}\left(P_{0}, L_{0}\right)$, but with a twist. Indeed, this twist is the end result of an isotopy carrying $P_{0}$ to $P_{m(t)}, 0 \leqslant t \leqslant 1$.

(ii) No generality is lost in choosing the base point of $M$

$$
0=(0,1,2, \ldots, n+1, n+2) \text {. }
$$

Then $\pi_{1}(M, 0)$ consists of $n+3$ paths $c_{i}(t)$ in $P, 0 \leqslant t \leqslant 1$, with (1) $c_{i}(0)=$ $c_{i}(1)=i, 0 \leqslant i \leqslant n+2$; (2) the "strings" $\left(c_{i}(t), t\right), 0 \leqslant t \leqslant 1$, in $P \times \mathbf{R}$ do not intersect. Thus $\pi_{1}(M, 0)$ is the colored braid group $C_{n+3}(P)$ on $n+3$ strings in $P$. The action of $\pi_{1}(M, 0)$ on $P H^{1}\left(P_{0}, L_{0}\right)$ is the action of the colored braid group, defined previously. Let the homomorphism

$$
\theta: \pi_{1}(M, 0) \rightarrow \text { Aut } P H^{1}\left(P_{0}, L_{0}\right):=\operatorname{PGL}\left(H^{1}\left(P_{0}, L_{0}\right)\right)
$$

denote the action of $\pi_{1}(M, 0)$.

Definition. $\Gamma_{\alpha}=$ Image $\theta$ is called the $\alpha$-hypergeometric monodromy group.

The following facts are proved in [3].

LEMMA 3.3. If $\alpha_{s} \neq 1$ for all $s \in S$, then

$$
H^{1}\left(P_{0}, L_{0}\right)=H_{c}^{1}\left(P_{0}, L_{0}\right) \text {. }
$$

Here $H_{c}^{*}$ denotes cohomology with compact supports. The dual assertion $H_{1}\left(P_{0}, \breve{L}_{0}\right)=H_{1}^{l f}\left(P_{0}, \breve{L}_{0}\right)$ for homology asserts that a locally finite 1 -cycle with coefficients in the dual $\breve{L}$ of $L$ is homologous to a compactly supported one. We can illustrate this as follows. The half-open interval $(0, p]$ is a closed 1-chain in the plane punctured at 0 . In the chain complex with local coefficients $\check{L}$, a small positive loop $c$ around 0 has $\partial c=\alpha_{0}^{-1} p-p$. Hence $\partial \lambda c=$ $\partial(0, p]$ for $\lambda^{-1}=\alpha_{0}^{-1}-1$ and in fact $(0, p]$ is homologous to $\lambda c$.

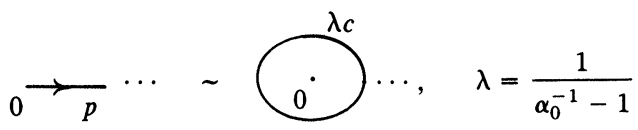

FIGURE 12

LEMMA 3.4. $\operatorname{dim} H^{1}\left(P_{0}, L_{0}\right)=n+1$ if $\alpha_{i} \neq 1$ for some $i \in\{0, \ldots, n+1\}$.
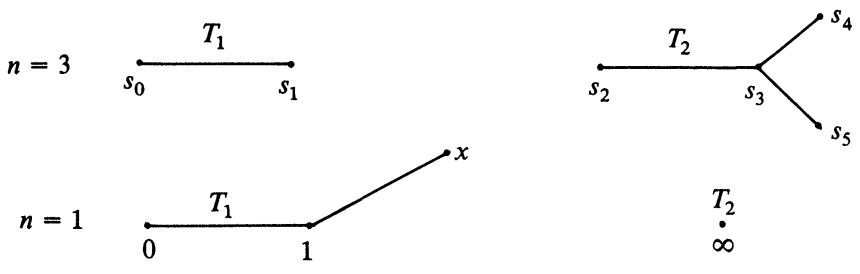

FIGURE 13 
A base for $H_{1}^{l f}\left(P_{0}, \check{L}_{0}\right)$, the dual of $H_{c}^{1}\left(P_{0}, L_{0}\right)$, can be illustrated. Set $S=P-P_{0}, \alpha_{s}=\alpha_{i}$ if $s=0(i)$. Partition the set of punctures $S$ into two (nonempty disjoint) subsets $S_{1}, S_{2}$. Choose two trees $T_{1}, T_{2}$ and an embedding $\beta$. $T_{1} \amalg T_{2} \rightarrow P$ with the vertices of $T_{i}$ going to $S_{i}(i=1,2)$. For each open edge $a$ of $T_{1}$ or $T_{2}$ choose an orientation of $a$ and a section $l(a) \in H^{0}\left(a \beta^{*} L_{0}\right)$. Then, as proved in [3],

LemmA 3.5. If $\Pi_{s \in S_{1}} \alpha_{s} \neq 1$, the set of 1-cycles $\left\{l(a) \beta \mid a\right.$; $\left.a \in T_{1} \cup T_{2}\right\}$ is a base of $H_{1}^{l f}\left(P-S, \check{L}_{0}\right)$.

From now on assume that

$$
\left|\alpha_{i}\right|=1 \text { for all } i, 0 \leqslant i \leqslant n+2 .
$$

Then the complex conjugate $\bar{L}$ of the flat one-dimensional bundle $L$ is isomorphic to $L$, the dual of $L$, and corresponds to $\alpha^{-1}=\left(\alpha_{0}^{-1}, \ldots, \alpha_{n+2}^{-1}\right)$. Consequently the monodromy group $\Gamma_{\alpha}$, which respects the natural cup product pairing

$$
H^{1}\left(P_{0}, L_{0}\right) \times H_{c}^{1}\left(P_{0}, \check{L}_{0}\right) \rightarrow H_{c}^{2}\left(P_{0}, \mathrm{C}\right) \simeq \mathrm{C},
$$

preserves a hermitian form

$$
H^{1}\left(P_{0}, L_{0}\right) \times \overline{H^{1}\left(P_{0}, L_{0}\right)} \rightarrow \mathrm{C},
$$

in view of Lemma 1 . Let $\psi$ denote the hermitian form on $H^{1}\left(P_{0}, L_{0}\right)$ defined by (3.7). Then we conclude

$$
\Gamma_{\alpha} \subset \operatorname{PU}\left(H^{1}\left(P_{0}, L_{0}\right), \psi\right)
$$

that is, $\Gamma_{\alpha}$ lies in the image in $\operatorname{PGL}\left(H^{1}\left(P_{0}, L_{0}\right)\right)$ of the unitary group of the hermitian form $\psi$. The hermitian form is unique up to a real scalar factor. One can normalize the choice of $\psi$ by imposing the condition: For any holomorphic 1-form $\omega$ on $P_{0}$ with coefficients in $L_{0}$,

$$
\psi([\omega],[\omega])=\int_{P_{0}} \int_{P_{0}} \omega \wedge \bar{\omega} \geqslant 0
$$

where $[\omega]$ is the cohomology class determined by the de Rham homomorphism.

LEMMA 3.8. Write $\alpha_{i}=e^{2 \pi \sqrt{-1}} \mu_{i}$ with $0 \leqslant \mu_{i}<1,0 \leqslant i \leqslant n+2$. Then the signature of the hermitian form is

$$
\left(\left(\sum_{i=0}^{n+2} \mu_{i}\right)-1, \sum_{i=0}^{n+2}\left(1-\mu_{i}\right)\right) \text {. }
$$

With $\alpha$ and $\mu$ related via $\alpha_{i}=e^{2 \pi \sqrt{-1} \mu_{i}}$ for each $i$, we set

$$
\Gamma_{\mu}=\Gamma_{\alpha} \text {. }
$$

3.9. We now relate the foregoing to hypergeometric functions of $n$ variables, which are by definition related to the differential form

$$
\prod_{i=0}^{n+1}\left(z-x_{i}\right)^{-\mu_{i}} d z
$$


where $\left(x_{0}, \ldots, x_{n+1}\right)$ and $\mu=\left(\mu_{0}, \ldots, \mu_{n+1}\right)$ are in $\mathbf{C}^{n+2}$. Define $\mu_{n+2}$ by

$$
\sum_{0}^{n+2} \mu_{i}=2 \text {. }
$$

Set $\alpha_{i}=e^{2 \pi \sqrt{-1} i}, 0 \leqslant i \leqslant n+2$, and let $L$ be a flat one-dimensional vector bundle on $P_{M}$ with monodromy $\alpha$ along each $P_{m}, m \in M$.

Let $e$ denote a multivalued section of $P_{m}$ in $L_{m}$; that is, a constant section of the product bundle $\mathbf{C} \times \hat{P}_{m}$ over the simply connected $\hat{P}_{m}$ viewed as an $L_{m}$-valued section on $P_{m}$. Then set

$$
\omega_{\mu}(m)=\prod_{i=0}^{n+1}\left(z-x_{i}\right)^{-\mu_{i}} e d z .
$$

This defines a meromorphic differential 1-form on $P$, holomorphic on $P_{m}$, whose "order" or "valuation" at $\infty$ is $-\mu_{n+2}$.

Set $M_{\infty}=\left\{\left(x_{0}, x_{1}, \ldots, x_{n+2}\right) \in M ; x_{n+2}=\infty\right\}$.

LEMMA 3.10. The cohomology class of $\omega_{\mu}(m)$ in $H^{1}\left(P_{m}, L_{m}\right)$ is nonzero.

LEMMA 3.11. The map $m \rightarrow w_{\mu}(m), m \in M_{\infty}$, defines a holomorphic section of $M$ in the flat projective space bundle $B(\alpha)_{M}$.

The group Aut $P$, which is just PGL(2), operates diagonally on $P^{n+3}$ and hence on $M$. Set

$$
Q=\text { Aut } P \backslash M=(\text { Aut } P)_{\infty} \backslash M_{\infty}
$$

where (Aut $P)_{\infty}$ is the subgroup of Aut $P$ fixing the point $\infty$. The fibering (3.2.1) $B(\alpha)_{M} \rightarrow M$ descends to $B(\alpha)_{Q} \rightarrow Q$. Up to a scalar factor, the value of (3.9.1) remains unchanged under the action of (Aut $B)_{\infty}$ so that the section of Lemma $3.11 \omega_{\mu}: M \rightarrow B(\alpha)_{M}$ descends to a well-defined section, also denoted $\omega_{\mu}$;

$$
\omega_{\mu}: Q \rightarrow B(\alpha)_{Q}
$$

We call this map the Schwarz section.

Choose a base point $o \in Q$, and above it $\hat{o} \in \hat{Q}$, the simply connected covering space of $Q$. Then the homomorphism (3.2.2) descends to a homomorphism

$$
\theta: \pi_{1}(Q, 0) \rightarrow \text { Aut } P H^{1}\left(P_{0}, L_{0}\right) .
$$

Schwarz section $w_{\mu}$ defines a $\pi_{1}(Q, 0)$-equivariant map

$$
\hat{w}_{\mu}: \hat{Q} \rightarrow P H^{1}\left(P_{0}, L_{0}\right) \text {. }
$$

In the case $n=1$, the map $\hat{w}_{\mu}$ is precisely Schwarz's multivalued map $P-\{0,1, \infty\} \rightarrow P$. Indeed, when $n=1$, Aut $P \backslash M=P-\{0,1, \infty\}$, we can write any point $m$ of $M_{\infty}$ as $(0,1, x, \infty) \bmod (\text { Aut } P)_{\infty}$, and choose as a base of linear functions on $H^{1}\left(P_{0}, L_{0}\right)$ the line segments $(0,1)(1, x)$, we find as homogeneous coordinates of $\hat{\omega}_{\mu}(x)$ $(3.11 .4)^{\prime}$

$$
\left(\int_{0}^{1} z^{-\mu_{0}}(z-1)^{-\mu_{1}}(z-x)^{-\mu_{2}} d z, \quad \int_{1}^{x} z^{-\mu_{0}}(z-1)^{-\mu_{1}}(z-x)^{-\mu_{2}} d z\right) .
$$


In the general case, the homogeneous coordinates of the map $\hat{\omega}_{\mu}$ are given by hypergeometric functions of $n$ variables. We call the map $\hat{\omega}_{\mu}$ of (3.11.4) the $S c h w a r z$ multivalued map when it is regarded as a map of the space $Q$.

3.12. Inasmuch as we are ultimately interested in constructing lattices in $\mathrm{PU}(1, n)$, we shall assume henceforth that

$$
\left\{\begin{array}{l}
0<\mu_{i}<1 \quad \text { for all } i=0,1, \ldots, n+2 \\
\sum_{i} \mu_{i}=2
\end{array}\right.
$$

It follows at once from Lemma 3.8 that the signature of the hermitian form is $(1, n)$.

In the case $n=1$, the signature of the invariant hermitian form explains why there is a common circle orthogonal to the circular arcs of Schwarz's triangle when $\lambda_{0}+\lambda_{1}+\lambda_{\infty}<1$.

An interesting interpretation of the Schwarz section results from the

LEMMA 3.13. $H^{1}\left(P_{0}, L_{0}\right)=H^{1,0}+H^{0,1}$ (direct).

Here $H^{1,0}$ is the subspace represented by holomorphic 1-forms on $P_{0}$, and $H^{0,1}$ is the subspace represented by anti-holomorphic 1 -forms, i.e., every cohomology class in $H^{0,1}$ is represented by an $L_{0}$-valued 1 -form $\omega$ with $\bar{\omega}$ a holomorphic $\bar{L}_{0}$-valued form on $P_{0}$.

Inasmuch as $P_{m}$ is unchanged topologically as $m$ varies, the fiber bundle $P_{M} \rightarrow M$ of (3.2) is topologically flat but not holomorphically for $n \geqslant 1$, since the holomorphy structure of $P_{m}$ does vary with $m$. Upon identifying $H^{1}\left(P_{m}, L_{m}\right)$ with $H^{1}\left(P_{0}, L_{0}\right)$ for $m$ near 0 , the subspace $H^{1,0}\left(P_{m}, L_{m}\right)$ varies in $H^{1}\left(P_{0}, L_{0}\right)$ as $m$ varies in $M$. By Lemma 3.8,

$$
\operatorname{dim} H^{1,0}=1 \text {. }
$$

Hence in $P H^{1}\left(P_{0}, L_{0}\right), P H^{1,0}\left(P_{m}, L_{m}\right)$ is a point. The Schwarz section of (3.11.1),

$$
\omega_{\mu}: Q \rightarrow P H^{1}\left(P_{0}, L_{0}\right),
$$

thus expresses the variation of holomorphic structure on $P_{q}$ as $q$ varies in $Q$.

3.14. In the 1885 paper [13] cited above, Picard generalized Schwarz's second problem: If $\lambda_{0}+\lambda_{1}+\lambda_{\infty}<1$ and $\lambda_{s}^{-1}$ is an integer $(s=0,1, \infty)$ then the Schwarz multivalued map

$$
\hat{\omega}_{\mu}: P-\{0,1, \infty\} \rightarrow P
$$

has a single-valued inverse $B^{1} \rightarrow P$.

Expressed in our notation, Picard asserted the following theorem.

Assume

$$
\begin{gathered}
0<\mu_{i}<1 \quad(i=0, \ldots, 4), \\
\sum_{i=0}^{4} \mu_{i}=2 .
\end{gathered}
$$

Then the Schwarz multivalued map

$$
\hat{\omega}_{\mu}: Q \rightarrow \text { complex projective 2-space }
$$


carries $\hat{Q}$ into the 2-ball $B^{2}$. If, moreover,

$$
\begin{gathered}
\mu_{i}+\mu_{j} \leqslant 1 \text { for all } 0 \leqslant i \neq j \leqslant 4, \\
\left(1-\mu_{i}-\mu_{j}\right)^{-1} \in \mathbf{Z} \cup \infty,
\end{gathered}
$$

then the map $\hat{w}_{\mu}$ has a single-valued inverse.

This result implies immediately that the monodromy group $\Gamma_{\mu}$ is discrete in PU(1,2), the subgroup of $\operatorname{PGL}(3, \mathbf{C})$ which keeps the complex 2-ball $B^{2}$ invariant. Furthermore, Picard could show that $\Gamma_{\mu} \backslash B^{2}$ has finite measure, which implies that $\Gamma_{\mu} \backslash \mathrm{PU}(1,2)$ has finite Haar measure.

Whereas all the claims of Picard are correct, his proof that $\hat{w}_{\mu}$ maps a slit simply connected domain in $Q$ to a fundamental domain of $\Gamma_{\mu}$ is defective (cf. [9]). In 1891 Picard remarked without proof that hypothesis (3.14.3) could be dropped.

In an effort to give a correct proof of Picard's theorem above and to generalize it to $n$ dimensions, Deligne and I were led to reformulate Picard's result, as follows.

THEOREM 3.15. Let $\mu=\left(\mu_{0}, \mu_{1}, \ldots, \mu_{n+2}\right), n \geqslant 1$.

Assume $\mu$ satisfies (3.14.1), (3.14.2), and the condition

$$
\begin{gathered}
\text { For all } 0 \leqslant i \neq j \leqslant n+2 \text {, such that } \mu_{i}+\mu_{j} \leqslant 1, \\
\left(1-\mu_{i}-\mu_{j}\right)^{-1} \in \mathbf{Z} \cup \infty .
\end{gathered}
$$

Then the Schwarz multivalued map $\hat{w}_{\mu}$ induces a $\Gamma_{\mu}$ equivariant homeomorphism

$$
\tilde{Q}_{s t}^{\mu} \rightarrow B^{n},
$$

where $B^{n}$ is a ball in complex projective $n$-space and $\tilde{Q}_{s t}^{\mu}$ is a branched covering of a completion $Q_{s t}^{\mu}$ of $Q$ which depends on $\mu$. Moreover, $\Gamma_{\mu}$ is a lattice in $\operatorname{PU}(1, n)$.

A central point in the proof is the selection of the correct completion $Q_{s t}^{\mu}$ of $Q$; this completion is the same as the weighted completion occurring in Mumford's Geometric invariant theory $[12 ; 3, \S 4]$.

4. Schwarz's first problem in $\boldsymbol{n}$-dimensions.

4.1. A chronology of the monodromy of hypergeometric functions begins with Euler's investigation of the hypergeometric differential equation in 1778 [4], continues through Riemann's clarification in 1857 of the global nature of a multivalued hypergeometric function [15], Schwarz's 1873 paper [17], and Picard's 1885 paper [13]. However, the chronology of the results presented in the foregoing section had its origins in 1978 with my attempt to construct nonarithmetic lattices in $U(2,1)$, in order to explore the limits of validity in the case of R-rank 1 groups of the celebrated 1974 theorem of G. A. Margulis, Irreducible lattices in semisimple Lie groups of R-rank greater than 1 are arithmetic [6].

In 1966 V. S. Makarov [5] had constructed nonarithmetic lattices generated by reflections in faces of geodesic polyhedra in real hyperbolic 3-space, $\mathbf{R} h^{3}$, and in a series of papers starting in 1967 [18], E. B. Vinberg carried out a basic 
systematic study of lattices generated by reflections in the faces of a geodesic simplex in real hyperbolic $n$-space, $\mathbf{R} h^{n}$, finding nonarithmetic lattices only for $n \leqslant 5$.

Direct efforts to transfer Vinberg's results to complex hyperbolic $n$-space $\mathrm{C} h^{n}$ were obstructed by the fact that $\mathrm{C} h^{n}$ has no geodesic polyhedra for $n>1$ since any geodesic subspace of $\mathbf{C} h^{n}$ is a complex submanifold and has even dimension.

If one tries nevertheless to mimic algebraically in $\mathrm{Ch}^{n}$ the construction of subgroups in the isometry group $\mathrm{PU}(n, 1)$ of $\mathbf{C} h^{n}$, one is led to groups generated by complex reflections, but then one faces the problem of proving these groups are discrete. In the case of $\mathbf{R} h^{n}$, the geodesic simplex one begins with is a fundamental domain if its faces meet at a dihedral angle $\pi / n$ with $n$ an integer. In the case of $\mathrm{Ch}^{n}$, the fundamental domain, if it exists, is elusive.

At the 1978 Helsinki Congress I announced a construction of a family of subgroups

$$
\Gamma_{p, t} \quad(|t|<3(1 / 2-1 / p), p=3,4,5)
$$

in $\mathrm{U}(1,2)$ which are generated by complex reflections in $\mathbf{C h} h^{3}$, and a proof that for 17 values of $(p, t)$ with $t \geqslant 0, \Gamma(p, t)$ is a lattice and that 7 of these lattices are nonarithmetic (cf. [8]). A summary of the construction is given in [11, §2].

The complex $n$-ball $B^{n}$ is of course a model for complex hyperbolic $n$ space $\mathrm{Ch}^{n}$ and $\mathrm{PU}(1, n)$ is the connected component of the identity in its group of isometries. Furthermore,

(4.3) The monodromy groups $\Gamma_{\mu}$ defined in $\S 3$ above are generated by complex reflections of $\mathbf{C} h^{n}$.

One sees this easily with the help of Lemma 3.5. For, given any two elements $s_{0}, s_{1}$ of the set $S$ of $n+3$ punctures, one chooses a tree $T_{1}$ consisting of a single edge and an embedding $\beta$ as in Lemma 3.4. $\beta: T_{1} \cup T_{2} \rightarrow P$ with the vertices of $T_{1}$ going to $\left\{s_{0}, s_{1}\right\}$ and the vertices of $T_{2}$ going to $S-\left\{s_{0}, s_{1}\right\}$.

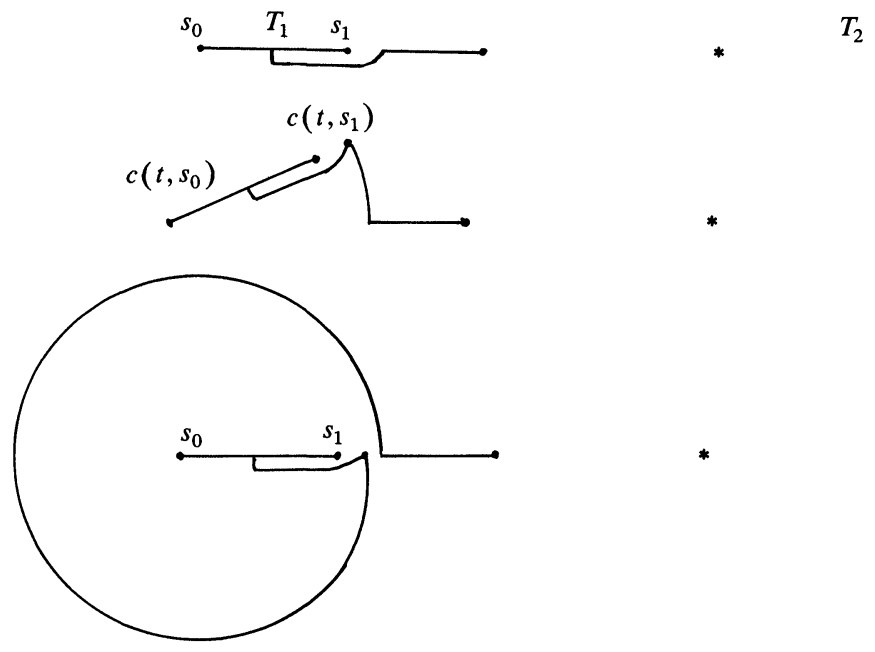

FIGURE 14 
One can then choose an isotopy twisting $s_{1}$ around $s_{0}$ which leaves fixed $\beta\left(T_{2}\right)$. Taking as base of $H_{1}^{l f}\left(P-S, \check{L}_{0}\right)$ the set of 1-cycles $\left\{l(a) \beta \mid a ; a \in T_{1}\right.$ $\left.\cup T_{2}\right\}, a$ denoting an oriented open edge and $l(a) \in H^{0}\left(a, \beta^{*} \breve{L}_{0}\right)$, we see from Figure 14 that for $v=l(a) \beta \mid a$

$$
v \rightarrow\left(\alpha_{0} \alpha_{1}\right)^{-1} v, \quad v \text { in } T_{1} ; \quad v \rightarrow v, \quad v \text { in } T_{2} .
$$

In 1980, when I first learned of the 1885 Picard theorem about lattices in PU(1,2) arising from monodromy of hypergeometric functions, the similarities between $\Gamma_{\mu}$ and $\Gamma_{p, t}$ were striking. However, it was possible to prove:

(4.4) The lattices $\Gamma_{p, t}$ of (4.2) and the lattices $\Gamma_{\mu}$ which satisfy Picard's integrality conditions are different.

Nevertheless, there is a relation between $\Gamma_{\mu}$ and $\Gamma_{p, t}$, first given in Generalized Picard lattices arising from half-integral conditions [10], which we shall elaborate on in $\$ 5$.

Suppose that $S_{1} \subset S$ and all the $\left\{\mu_{s} ; s \in S_{1}\right\}$ are equal. Let $\Sigma$ denote the permutation group of the subset $S_{1}$. We can regard $\pi_{1}(Q / \Sigma)$ as a subgroup of $\pi_{1}(Q)$ and, as shown in [10], the homomorphism

$$
\theta: \pi_{1}(Q, 0) \rightarrow \text { Aut } P H^{1}\left(P_{0}, L_{0}\right)
$$

of (3.11.3) extends to a homomorphism

$$
\theta: \pi_{1}(Q / \Sigma) \rightarrow \text { Aut } P H^{1}\left(P_{0}, L_{0}\right) .
$$

Set $\Gamma_{\mu \Sigma}=$ Image $\theta_{\Sigma}$. Then

is exact.

$$
1 \rightarrow \Gamma_{\mu} \rightarrow \Gamma_{\mu \Sigma} \rightarrow \Sigma \rightarrow 1
$$

THEOREM 4.6. Assume that

$$
\begin{aligned}
& 0<\mu_{s}<1 \quad \text { for all } s \in S, \\
& \sum_{s} \mu_{s}=2,
\end{aligned}
$$

and in addition $\left(\mu_{s}\right)_{s \in S}$ satisfies the condition:

$\left(\Sigma\right.$ INT) For all $s, t \in S$ with $\mu_{s}+\mu_{t}<1$,

$$
1-\mu_{s}-\mu_{t} \in \begin{cases}\frac{1}{2} \mathbf{Z} & \text { if } s, t \in S_{1}, \\ \mathbf{Z} & \text { otherwise. }\end{cases}
$$

Then $\Gamma_{\mu}$ is a lattice in $\mathrm{PU}(1, n), n=\operatorname{card} S-3$.

REMARK 1. Inasmuch as $\Gamma_{\mu \Sigma}$ is a finite extension of $\Gamma_{\mu}, \Gamma_{\mu \Sigma}$ is a lattice whenever $\Gamma_{\mu}$ is.

REMARK 2. We will see in the next section that the subgroups $\Gamma_{p, t}$ of (4.2) are conjugate in $\mathrm{PU}(1,2)$ to a subgroup of $\Gamma_{\mu \Sigma}$ of index at most three. Chronologically, this is the observation that led to the investigation of condition ( $\Sigma$ INT).

The next theorem asserts the converse of Theorem 4.5 for dimensions $n>3$.

THEOREM 4.7. Assume $n>2$ and

$$
\begin{aligned}
& 0<\mu_{0}<1 \quad \text { for all } 0 \in S, \\
& \sum_{s} \mu_{s}=2 .
\end{aligned}
$$

If $\Gamma_{\mu}$ is discrete in $\mathrm{PU}(1, n)$, then $\mu=\left\{\mu_{s}\right\}_{s \in S}$ satisfies condition ( $\Sigma$ INT) except for $\mu=\left(\frac{1}{12}, \frac{3}{12}, \frac{5}{12}, \frac{5}{12}, \frac{5}{12}, \frac{5}{12}\right)$. 
This theorem is proved in [11b]. (Note the error in the hypothesis of Theorem 4 of [11a]: $d>3$ not $d>1$; cf. [11b].) Theorems 4.6 and 4.7 solve Schwarz's first problem for the monodromy groups fixing a hermitian form of signature $(1, n)$. In the case that $2<\Sigma_{s} \mu_{s}<n$, set $p=\left(\Sigma_{s} \mu_{s}\right)-1$ and $q=n+1-p$. Then $\Gamma_{\mu} \subset \mathrm{PU}(p, q)$ by Lemma 3.8. The condition that $\Gamma_{\mu}$ be closed in $\mathrm{PU}(p, q)$ implies that (as in the case of $\mathrm{PU}(1, n)) \Gamma_{\mu}$ is a lattice in $\operatorname{PU}(p, q)$. Therefore, by the celebrated theorem of G. A. Margulis [6], $\Gamma_{\mu}$ is an arithmetic lattice in $\operatorname{PU}(p, q)$. On the other hand, in Proposition 12.7 of [3], there is a criterion for $\Gamma_{\mu}$ to be arithmetic in $\operatorname{PU}\left(H^{1}\left(P_{0}, L_{0}\right), \psi\right), \psi$ denoting the invariant hermitian form, that is valid for any signature $(p, q)$ :

(4.8) Let $d$ denote the least common denominator of $\left\{\mu_{s}: s \in S\right\}$. Then $\Gamma_{\mu}$ is a lattice in $\mathrm{PU}\left(H^{1}\left(P_{0}, L_{0}\right)\right)$ if and only if for each integer $A$ relatively prime to $d$, with $1<A<d-1$,

$$
\sum_{s \in S}\left\langle A \mu_{s}\right\rangle=1 \text { or } \quad \operatorname{card} S-1 .
$$

Here for any rational number $b,\langle b\rangle$ denotes the fractional part of $b$, i.e. 0 $\leqslant\langle b\rangle\langle 1$ and $b-\langle b\rangle \in \mathbf{Z}$.

Theorem 4.7 and (4.8) together yield all the $\mu$ such that $\Gamma_{\mu}$ is discrete in all cases that $n>2$. In the case that $p=0$ or $q=0, \Gamma_{\mu}$ is closed if and only if it is finite, and then the condition in (4.8) is satisfied. In the case $n \leqslant 2$, neither the condition ( $\Sigma$ INT) nor the condition (4.8) is necessary if the group $\Gamma_{\mu}$ is infinite. The list of all lattices $\Gamma_{\mu}$ in $\mathrm{PU}(1, n)$ not satisfying $(\Sigma$ INT) is derived in [11b]. That list together with (4.8) and Theorem 4.7 give a complete solution of Schwarz's first problem.

5. Presentations for $\Gamma_{\mu}, \Gamma_{(p, t)}$, and $\Gamma_{\mu \Sigma}$. In order to describe explicitly the relation between the $\Gamma_{p, t}$ and the $\Gamma_{\mu \Sigma}$, it is convenient to choose a set of generators for the pure braid group on 5 strings in $\mathbf{P}^{1}$ that is stable under the permutation group of the subset $S_{1}$ of punctures $S=\left\{s_{0}, s_{1}, s_{2}, s_{3}, s_{4}\right\}$.

Assume $S_{1}=\left\{s_{0}, s_{1}, s_{2}\right\}$ and assume $\mu_{0}=\mu_{1}=\mu_{2}$.

Identify the projective line $P$ with $S^{2}$, the 2-sphere with its standard metric. Choose $s_{0}, s_{1}, s_{2}$ equally spaced on the equator of $S^{2}$ with $s_{3}$ and $s_{4}$ at the North and South poles respectively. Denote by $i j$ for any $i \neq j$ with $i, j \in\{0,1,2,3,4\}$ the pure braid that moves $s_{i}$ along the shortest path to a point near $s_{j}$, then makes a small circuit in the positive sense around $s_{j}$, and then returns to its original position. For $i, j \in\{0,1,2\}$. Let $i \hat{j}$ denote the braid that interchanges $i$ and $j$ via a half-turn isotopy in the positive sense that leaves each point fixed outside of a small neighborhood of the shortest arc joining $i$ to $j$.

Let $J$ denote the cyclic permutation $0 \rightarrow 1 \rightarrow 2 \rightarrow 0$ of $\{0,1,2,3,4\}$. We denote by $J$ also its realization as a rotation by angle $2 \pi / 3$ in the positive sense around the North pole of $P$, its effect on $P H^{1}(P-S, L)$, and its realization as a braid in $B_{5}(P)$, the braid group on 5 -strings in $P$. We shall require below the following braid group identities that can be verified directly from inspection of the diagrams (see Figure 15).

$$
\begin{aligned}
& J^{-1} 0112=(23)^{-1} \\
& J 1201=04)^{-1} \\
& J 12=20 J
\end{aligned}
$$




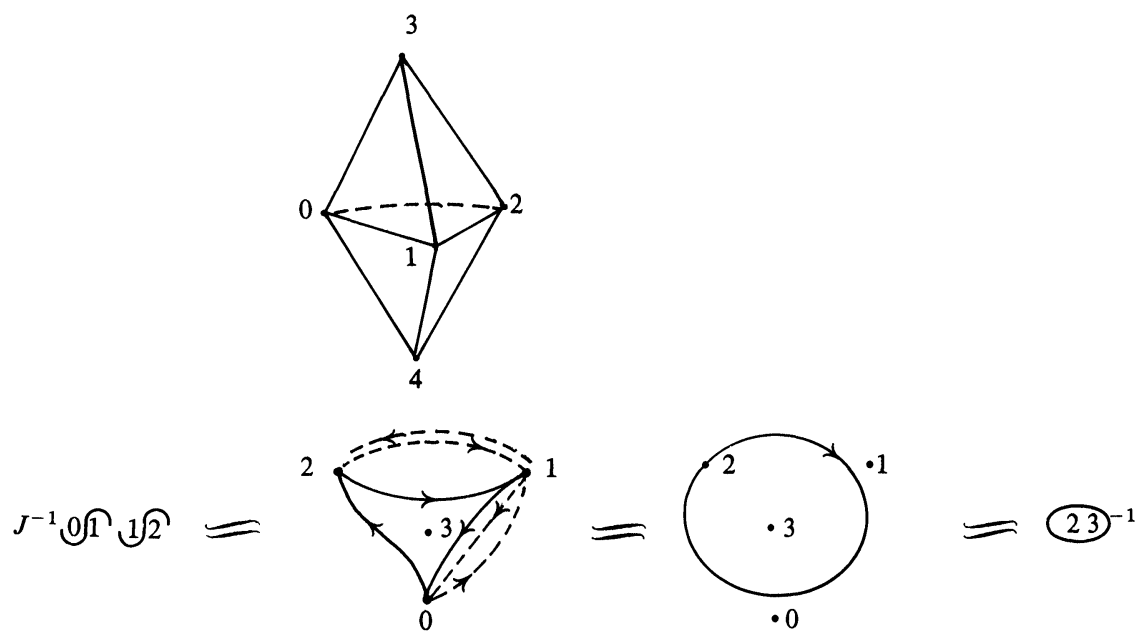

Figure 15

Set

$$
\begin{aligned}
& A_{i}=\theta(3 i), \quad A_{i}^{\prime}=\theta(40), \\
& B_{i}=\theta((i-1 i+1)), \quad i=0,1,2(\bmod 3) \\
& R_{i}=\theta(i-1 \sqrt{i+1}) \\
& B_{i}^{\prime}=\theta\left(34_{i}\right),
\end{aligned}
$$

where the circuit 34 is chosen so as to cross the equator only on the short arc $(i-1, i+1)$.

From the braid identities (5.1), we infer

$$
\begin{gathered}
J^{-1} R_{i} R_{i+1}=A_{i}^{-1}, \quad J R_{i+1} R_{i}=A_{i+1}^{\prime-1}, \\
J R_{i}=R_{i+1} J, \quad i=0,1,2(\bmod 3) .
\end{gathered}
$$

The product of the pure braids

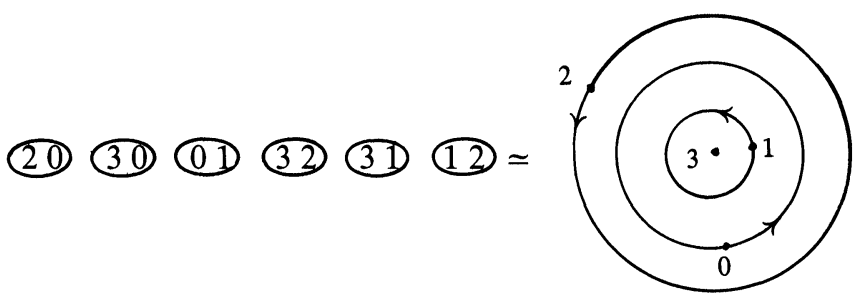

FIGURE 16

is in the center of the colored braid group $C_{4}\left(\mathbf{R}^{2}\right)$ on 4-strings in $\mathbf{R}^{2}$, and therefore its image in $\Gamma_{\mu}$ is central in $\Gamma_{\mu}$, and therefore central in $\operatorname{PU}(1,2)$ since $\Gamma_{\mu}$ is of finite covolume in $\mathrm{PU}(1,2)$, by a well-known (cf. [2]) result of $\mathrm{A}$. Selberg. Inasmuch as $\mathrm{PU}(1, n)$ has only the identity element in its center, we 
get

$$
B_{1} A_{0} A_{2} B_{2} A_{1} B_{0}=1 .
$$

The group $\Gamma_{\mu}$ is generated by any five of $\left\{A_{0}, A_{1}, A_{2}, B_{0}, B_{1}, B_{2}\right\}$.

As additional identities coming from the braid group we get

$$
\begin{aligned}
& A_{i}^{\prime}=A_{i-1} A_{i+1} B_{i}=B_{i} A_{i-1} A_{i+1}=A_{i+1} B_{i} A_{i-1}, \\
& B_{i}^{\prime}=A_{i-1}^{-1} A_{i}^{-1} A_{i+1}^{-1}=A_{i+1}^{\prime-1} A_{i}^{\prime-1} A_{i-1}^{\prime-1}=A_{i-1}^{-1} B_{i-1} A_{i-1}^{\prime-1}=A_{i+1}^{\prime} B_{i+1} A_{i+1}^{-1} \\
& \quad=\left(R_{i-1} R_{i+1}\right)^{3}, \\
& B_{i}=R_{i}^{2}, \\
& A_{i} B_{i}=B_{i} A_{i}, \\
& A_{i} A_{j}^{\prime}=A_{j}^{\prime} A_{i} \text { for } j \neq i, \\
& B_{i} B_{j}^{\prime}=B_{j}^{\prime} B_{i} \text { for } j \neq i .
\end{aligned}
$$

For any $i, j$ with $i \neq j$, set

$$
k_{i j}=\left(1-\mu_{i}-\mu_{j}\right)^{-1}
$$

We assume henceforth that $\mu$ satisfies condition ( $\Sigma$ INT) for $S_{1}$. Then $k_{i j}$ is an integer except when $i, j \in\{0,1,2\}$. For any $i \neq j \in\{0,1,2\}$ we set

$$
\begin{aligned}
k & = \begin{cases}k_{i j} & \text { if } k_{i j} \in \mathbf{Z}, \\
2 k_{i j} & \text { otherwise, }\end{cases} \\
k_{3} & =k_{3 i}, \quad k_{4}=k_{4 i} \quad(i, j=0,1,2) .
\end{aligned}
$$

Then

$$
\Gamma_{\mu} \text { has the presentation }
$$

Generators: $\quad A_{1}, A_{2}, A_{0}, B_{1}, B_{2}, B_{0}$

Relations: $\quad A_{i} B_{i}=B_{i} A_{i}, \quad B_{1} A_{0} A_{2} B_{2} A_{1} B_{0}=1$,

$$
\begin{aligned}
& A_{i}^{k_{3}}=1, \quad B_{i}^{k}=1, \\
& \left(A_{i-1} A_{i+1} B_{i}\right)^{k_{4}}=1, \quad\left(A_{1}^{-1} A_{2}^{-1} A_{0}^{-1}\right)^{k_{34}}=1 .
\end{aligned}
$$

The group $\Gamma_{\mu \Sigma}$ has the additional generators $R_{0}, R_{1}, R_{2}$. In view of (5.3), the group $\Gamma_{\mu}$ can be generated by $J$ and $R_{0}$ only, and from (5.5) and (5.6) one can derive a presentation for $\Gamma_{\mu \Sigma}$. has

Set $\Gamma_{\mu}^{*}=\left\langle R_{0}, R_{1}, R_{2}\right\rangle$, the subgroup of $\Gamma_{\mu \Sigma}$ generated by $R_{0}, R_{1}, R_{2}$. One

$$
\begin{aligned}
\left(R_{0} R_{1} R_{2}\right)^{3} & =J^{3} \cdot R_{0} R_{1} \cdot R_{2} R_{0} \cdot R_{1} R_{2}=J^{2} R_{1} R_{2} \cdot J R_{2} R_{0} \cdot R_{1} R_{2} \\
& =A_{1}^{-1} \cdot J^{-2} R_{2} R_{0} \cdot R_{1} R_{2}=A_{1}^{-1} J^{-1} R_{1} R_{2} \cdot J^{-1} R_{1} R_{2} \\
& =A_{1}^{-3}
\end{aligned}
$$

Hence

$$
\left(R_{0} R_{1} R_{2}\right)^{3 k_{3}}=A_{1}^{-3 k_{3}}=1
$$


Similarly

$$
\left(R_{2} R_{1} R_{0}\right)^{3 k_{4}}=A_{1}^{\prime-3 k_{4}}=1
$$

and from (5.5) one deduces

$$
\left(R_{0} R_{2}\right)^{3 k_{34}}=1 \text {. }
$$

Relations (a)-(c) yield a presentation for $\Gamma_{\mu}^{*}$ which coincides with the presentation for $\Gamma_{p, t}$ given in [8] when we take $(p, t)$ and $\mu$ related by

$$
\begin{aligned}
& \mu_{0}=\mu_{1}=\mu_{2}=\frac{1}{2}-\frac{1}{p}, \\
& \mu_{3}=\frac{1}{4}+\frac{3}{2 p}-\frac{1}{2} t, \quad \mu_{4}=\frac{1}{4}+\frac{3}{2 p}+\frac{1}{2} t,
\end{aligned}
$$

that is,

$$
p=\left(\frac{1}{2}-\mu_{0}\right)^{-1}, \quad t=\mu_{3}-\mu_{4} .
$$

By the strong rigidity theorem for $\mathrm{PU}(1, n), n>1$ we conclude that [7] $\Gamma_{\mu}^{*}$ is conjugate in $\mathrm{PU}(1,2)$ to $\Gamma_{p, t}$. Thus we conclude

THEOREM 5.8. The lattices $\Gamma_{p, t}$ of $(4.2)$ are conjugate in $\mathrm{PU}(1,2)$ to the subgroup $\Gamma_{\mu}^{*}$ of $\Gamma_{\mu \Sigma}$, where $\mu_{0}=\mu_{1}=\mu_{2}=\frac{1}{2}-\frac{1}{p}, \mu_{3}=\frac{1}{4}+\frac{3}{2 p}-\frac{1}{2} t, \mu_{4}=\frac{1}{4}$ $+\frac{3}{2 p}+\frac{1}{2} t$. The lattice $\Gamma_{p, t}$ is conjugate to a subgroup of index at most 3 in $\Gamma_{\mu \Sigma}$. In any case, $\Gamma_{\mu \Sigma} \simeq\left\langle J, \Gamma_{p, t}\right\rangle$.

In $\$ 17.1$ of [8], there is a criterion for deciding whether or not $J$ belongs to $\Gamma_{p, t}$. For the 17 lattices $\Gamma_{p, t}$ with $p=3,4,5$, the element $J$ belongs to $\Gamma_{p, t}$ in 7 cases, and for those cases $\Gamma_{\mu \Sigma}=\Gamma_{p, t}$. In those 7 cases, $\Gamma_{\mu}=\Gamma_{p, t}$ for $p=3,5$ but the $\mu$ does not satisfy the condition (INT) of Theorem 3.15; for $p=4, \mu$ satisfies condition (INT) and $\Gamma_{\mu}$ is of index 6 in $\Gamma_{p, t}$.

In elaboration of remark (4.4) above, there are 27 solutions $\mu$ satisfying the hypotheses of Theorem 3.15 for $n=2$, ignoring permutations of indices (cf. [3]). Of these 27 5-tuples, 22 have three equal terms (cf. [4]) and for such $\mu$, the lattice $\Gamma_{\mu}$ is commensurable after conjugacy with $\Gamma_{p, t}$; in these 22 cases, the integers $p$ are even. Thus, under the isomorphisms implicit in (5.7), the list of Picard lattices omits the $\Gamma_{\mu}$ with $\mu$ satisfying ( $\Sigma$ INT) but not (INT) and among these are, even allowing for commensurability, the $\Gamma_{p, t}$ with $p$ odd.

\section{BIBLIOGRAPHY}

1. E. Artin, Theorie der Zöpfe, Abh. Math. Sem. Hamburg Univ. 4 (1925), 42-72; Theory of braids, Ann. of Math. (2) 48 (1947), 101-126.

2. A. Borel, Density properties for certain subgroups of semi-simple groups without compact components, Ann. of Math. (2) 72 (1960), 179-188.

3. P. Deligne and G. D. Mostow, Monodromy of hypergeometric functions and non-lattice integral monodromy, Inst. Hautes Études Sci. Publ. Math. ${ }^{\circ} 63$ (1986).

4. L. Euler, Specimen transformationi singularis serierum, Sept. 3, 1778, Nova Acta Petropolitana, Bd XII (1801), 58-78.

5. V. S. Makarov, On a class of discrete subgroups in Lobachevsky space having an unbounded fundamental domain of finite measure, Dokl. Akad. Nauk SSSR 167 (1966), 30-33.

6. G. A. Margulis, Discrete groups of motions of manifolds of non-positive curvature, Proc. Int. Congr. Math., Vancouver (1974).

7. G. D. Mostow, Strong rigidity of locally symmetric spaces, Ann. Math. Studies, vol. 78, Princeton Univ. Press, Princeton, N. J., 1973. 
8. On a remarkable class of polyhedra in complex hyperbolic space, Pacific J. Math. 86 (1980), 171-276.

9. 78 (1981), 5948-5950.

10. Generalized Picard lattices arising from half-integral conditions, Inst. Hautes Études Sci. Publ. Math. ${ }^{\circ} 63$ (1986).

11a. L Lattices in $U(n, 1)$ (to appear).

b. appear).

12. D. Mumford, Geometric invariant theory, Springer

13. E. Picard, Sur les fonctions hyperfuchsiennes provenant des séries hypergéometrique de deux variables, Ann. ENS III 2 (1885), pp. 357-384.

14. L. Pochhammer, Über hypergeometrische Function hoheren Ordnung, J. für Math. 71 (1870), 316-362.

15. B. Riemann, Abh. Kon. Ges. Wiss. Göttingen VII (1957).

16. L. Schläfli, Über die Gaussiche hypergeometrische Reihe, Math. Ann. III (1871), 286-295.

17. H. A. Schwarz, Über diejenige Fälle in welchen die Gaussische hypergeometrische Reihe eine algebraische Function ihres viertes elementes darstellt, Crelle's J. 75 (1873), 292-335.

18. E. B. Vinberg, Discrete groups generated by reflections in Lobachevski spaces, Mat. Sb. (NS) 72 (114) (1967), 471-488.

Department of Mathematics, Yale University, New Haven, Connecticut 06520 